\title{
ENSAIO CLÍNICO COM MEBENDAZOLE NAS TENÍASES *
}

\author{
Naftale Katz e Fábio Zicker
}

\begin{abstract}
Foram tratados 31 pacientes com teníases pelo mebendazole. Utilizando-se os esquemas de $100 \mathrm{mg}$, duas vezes ao dia, por 4 dias consecutivos, $200 \mathrm{mg}$, duas vezes ao dia por 2 e $200 \mathrm{mg}$, duas vezes ao dia, por 4 dias, o percentual de cura foi de respectivamente $20,0,72,7$ e 90,0\%. Não houve diferença significativa quando foram tratados pacientes com Taenia solium $e$ Taenia saginata. $A$ ausência de efeitos colaterais e a ampla atividade anti-helmintica do mebendazole, recomendam seu uso também, como um novo agente tenicida.
\end{abstract}

\section{INTRODUÇÃO}

Ensaios clínicos com o mebendazole, realizados no Brasil e no exterior, mostraram que esta droga possue amplo espectro de ação em nematóides intestinais. De fato, Brugmans \& cols. (5), Chaia \& Cunha (7), Chaia \& cols. (8), Amato Neto \& cols. (3), Souza \& cols. (17), Katz (11) entre outros, demonstraram a eficácia terapêutica do mebendazole nas infecçōes por $A s-$ caris lumbricoides, Trichiuris trichiura, Enterobius vermicularis e em ancilostomídeos (Necator americanus e Ancylostoma duodenale).

O mebendazole age tanto "in vitro" como "in vivo", inibindo reaçōes metabólicas relacionadas com a captaçāo de glicose, produzindo uma depleção de glicogênio e um decréscimo na produção de ATP que é essencial para a sobrevida e reprodução dos parasitas (11).

Esta ação bloqueadora é responsável pelo amplo espectro da droga, permitindo agir simultaneamente sobre diferentes espécies parasitas na luz intestinal.

Baseado neste fato, foi realizado um ensaio clínico nas infecçōes por Taenia (so- lium e saginata) cujos resultados são apresentados no presente trabalho.

\section{MATERIAL E MÉTODOS}

Foram tratados 31 pacientes, sendo 10 masculinos e 21 femininos. A idade variou de 2 a 56 anos. Quanto à infecção, em 11 pacientes era ocasionada por Taenia solium, em 5 por $T$. saginata e em 13 não foi possível a identificação da espécie (Taenia $s p$ ).

Foram utilizados 3 esquemas terapêuticos:

Grupo $A-10$ pacientes tratados com $100 \mathrm{mg}$ duas vezes ao dia, durante 4 dias consecutivos.

Grupo $B-11$ pacientes com 200 $\mathrm{mg}$, duas vezes ao dia, durante 2 dias.

Grupo $C-10$ pacientes com $200 \mathrm{mg}$, duas vezes ao dia, durante 4 dias.

O medicamento foi sempre administrado pela manhã e à tarde, em regime ambulatorial e sem nenhuma restrição alimentar ou de atividades fisicas

Trabalho realizado no Centro de Pesquisas René Rachou - Instituto de Endemias Rurais e Seçăo de Parasitoses da Prefeitura de Belo Horizonte, com o auxilio do Conselho Nacional de Pesquisas.

Endereço: INERu - Caixa Postal, 1743 - 30.000 Eelo Horizonte. MG - Brasil.

Recebido para publicação em 13.4.72 

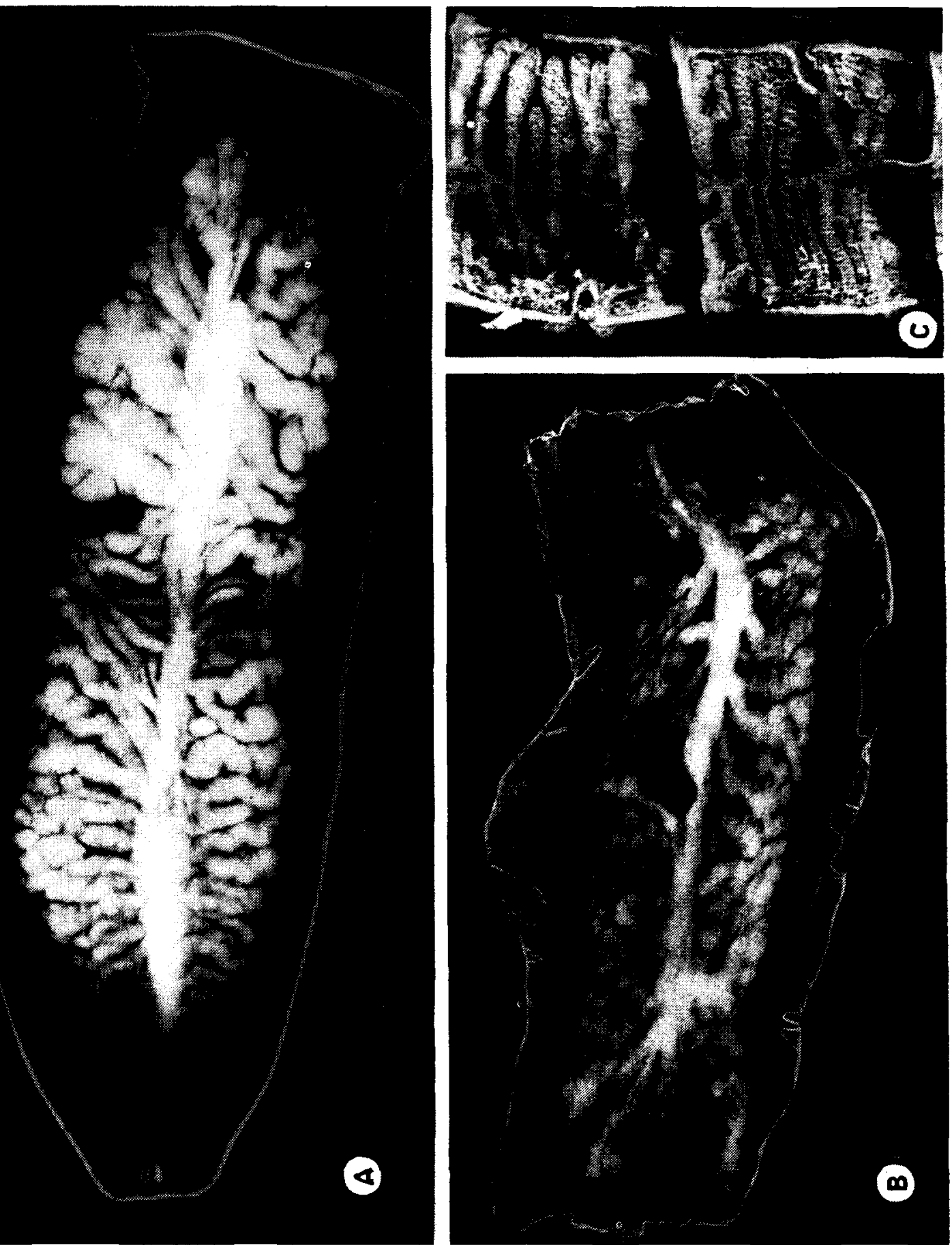

1.ig. I - Proglotes de Tcenia solium eliminadas com as fezes antes (A) e após o tratamento com mebendazole ( $\mathbf{B}$ e $C$ ). Nestas ultimas os sesmentos uterinos encontram-se alterades (aumento: $10.5 \times$ ). 
O diagnóstico parasitológico foi baseado no achado de ovos e/ou proglotes eliminados com as fezes.

o controle da atividade medicamentosa foi realizado através do exame das fezes eliminadas em 24 horas, recolhidas desde a primeiro dia de administração do mebendazole, até um dia após a última dose. As fezes foram emulsionadas com formol a $10 \%$, tamizadas e as proglotes encontradas comprimidas entre duas lâminas, clarificadas pelo ácido acético glacial e em seguida classificadas. Após 3 meses de tratamento, os pacientes foram perguntados se continuavam eliminando proglotes e novamente, fezes coletadas durante 24 horas, por dois dias sucessivos foram examinadas, à procura de proglotes (como acima descrito) e submetidas a exame parasitológico pelos métodos de Kato (14) e Hoffman, Pons \& Janer (10).

Os pacientes que a estes últimos exames não apresentaram, seja à anamnese ou ao exame das fezes, ovos e/ou proglotes de Taenia, foram considerados como curados.

\section{RESULTADOS}

A habela I mostra os resultados terapêuticos obtidos com os diferentes esquemas utilizados.

No Grupo A, tratado com o esquema usualmente recomendado para a cura de nematóides intestinais, o percentual de cura nos cestóides foi de apenas $20,0 \%$.
Já nos Grupos B e C, com doses duas e quatro vezes maiores que a utilizada no Grupo A, foi de $72,7 \%$ e $90,0 \%$, respectivamente. Com o esquema empregado no Grupo $\mathbf{C}$ não houve diferença no percentual de cura das $T$. solium e $T$. saginata.

Ressalte-se que em nenhum dos pacientes tratados encontrou-se o escolex. Por outro lado, frequientemente as proglotes eliminadas estavam alteradas, dificultando ou mesmo impedindo a sua classificação. $\mathrm{Na}$ Fig. 1 podem ser vistos algumas proglotes de $T$. solium eliminadas com as fezes antes (A) e após o tratamento ( $\mathrm{B}$ e $\mathbf{C}$ ). Nestas últimas, observa-se a ação da droga sobre os segmentos uterinos.

\section{DISCUSSÃO}

A ação do mebendazole nos pacientes poli-parasitados faz com que esta droga seja ímpar no arsenal terapéutico pois ao mesmo tempo apresenta elevada atividade nematocida e tenicida.

De fato, com dose total de $1600 \mathrm{mg}$ administrada em 4 dias, o percentual de cura nas teníases foi de $90 \%$. Este percentual obtido com mebendazole é semelhante àqueles descritos com a utilizaçāo da niclosamida [Rodrigues da Silva \& cols. (15), Amato Neto \& Campos (2), Khalil (13)], diclorofeno [Seaton (16), Cavier (6), Dufek \& Kalivoda (9)] e paromomicina [Botero (4), Wittner \& Tanowitz (18) 1 .

\title{
TABELA I
}

Atividade terapéutica do mebendazole $\mathrm{em}$ pacientes com teníases.

A - $100 \mathrm{mg}$ duas vezes ao dia $\times 4$ dias

$\mathrm{B}-200 \mathrm{mg}$ duas vezes ao dia $\times 2$ dias C - $200 \mathrm{mg}$ duas vezes ao dia $\times 4$ dias

No de pacientes tratados/ curados

\author{
Grupo e Esquema
}

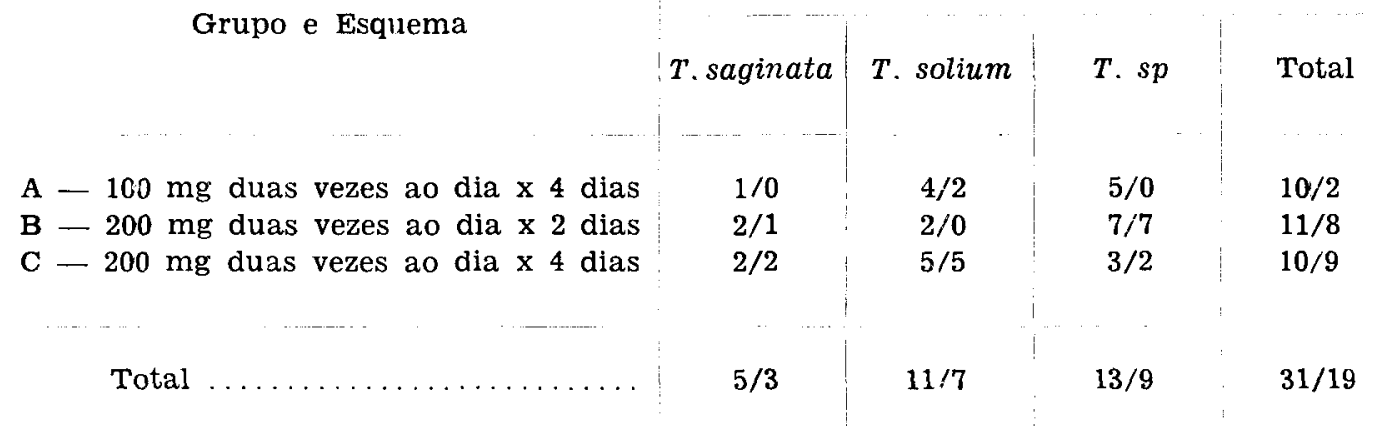


Estas drogas citadas são as que mais comumente têm sido utilizadas na clínica, devido à fácil administração e boa atividade terapêutica.

Fato a ser mencionado é o de não ter sido encontrado o escolex nas fezes dos pacientes tratados com mebendazole, em que pese a realização dos exames parasitológicos após 3 meses da terapêtica revelarem que os mesmos estavam curados. Vale mencionar a experiência de Ahkami \& Hadjian (1) que comprovaram que nos pacientes tratados com niclosamida, os escoleces eram detectados regularmente nas fezes se o tempo de exame das mesmas era menor que 7 horas, todavia, não eram mais encontrados se o exame era realizado 8 horas após a administração da droga. Este fato observado com o emprego da niclosamida deverá ser investigado com o mebendazole.

A ausência de efeitos colaterais, associada à ampla atividade anti-helmíntica do mebendazole, recomendam seu uso também como um novo agente tenicida.

\section{AGRADECIMENTOS}

Os autores agradecem aos técnicos Gercy de Souza Morais, Oswaldo de Souza Morais e Antônio Emídio Ferreira pela valiosa cooperação, bem como a Johnhon \& Johnson pelo fornecimento da droga e ao Sr. Joseph Wolf, do CPq. René Rachou, INERu, pela documentação fotográfica.

\section{SUMMARY}

31 patients with Taenia have been treated with mebendazole. With the schedules of $100 \mathrm{mg}$, bid, for 4 consecutive days, $200 \mathrm{mg}$, bid for 2 and $200 \mathrm{mg}$, bid for 4 , the percentages of cure were respectively, 20,0\%, 72,7\% and 90,0\%. No significant difference could be observed when patients with Taenia solium and Taenia saginata were treated with the most active schedule. The absence of side effects and the extensive anti-helminthic activity of mebendazole highly recommend its use inclusive as a new taenicide agent.

\section{REFERÊNCIAS BIBLIOGRÁFICAS}

1. AHKAMI, S. \& HADJIAN, A. - The appearance of the scolex of Taenia saginata in the stool after the eradication of the parasite by niclosamide. Ztschr. Tropenmed. Parasit., 20: 341-5, 1969 .

2. AMATO-NETO, V. \& CAMPOS, R. Tratamento por um derivado da salicilamida, de infestaçōes causadas por Taenia saginata e Taenia solium. Rev. Inst. Med. trop. São Paulo, 6: 297299,1964 .

3. AMATO-NETO, V., LEVI, G. C., STEFANI H. N. V., KONICHI, S. R., DIAS, J. C. P., OLIVEIRA, L. R. \& CAMPOS, L. L. - Nossas primeiras observações sobre a ação terapêtica do mebendazole, novo medicamento anti-helmíntico, dotado de amplo espectro de atividade. Rev. Inst. Med. trop. São Paulo, 15: 34-37, 1973.
4. BOTERO, R. D. - Paramomycin as effective treatment of Taenia infections. Amer. J. trop. Med. Hyg. 19: 234-237, 1970 .

5. BRUGMANS, J.P., THIENPONT, D.C., VAN WIJGAARDEN, L., VANPARIJS, O. F., SCHUERMANS, V. L. \& LAUWERS, H. L. - Mebendazole in enterobiasis. Radiochemical and pilot clinical study in 1.278 subjects. J. A. M. A., 217: 313-316, 1971.

6. CAVIER, R. - Les nouveaux médicaments utilisables dans le traitement du téniasis chez l'homme. Prod. Probl. pharm., 21: 581, 1966.

7. CHAIA, G. \& CUNHA, A. S. - Therapeutic action of mebendazole ( $R$ 17.635 ) against human helminthiasis. Folha Médica, 63: 843-852, 1971. 
8. CHAIA, G., MÉTENE, F., CHIARI, L., ARAUJO, S. M. \& ABREU, I. B. Mebendazole - um novo anti-helmíntico de ação terapêutica polivalente. Folha Médica, 64: 139-145, 1972.

9. DUFEK, M. \& KALIVODA, R. - Experience with modern treatment of taeniasis. Rev. Czech. Med., 15: 2932,1969 .

10. HOFFMAN, W. A., PONS, J. A. \& JANER, J. L. -- Sedimentation concentration method in Schistosomiasis mansoni. Puerto Rico J. Publ. Health. Trop. Med., 9: 283-298, 1934.

11. JOHNSON \& JOHNSON - Divisão Farmacêutica - Pantelmin (mebendazol). Documentação, 19 pp., 1972.

12. KATZ, N. - Anti-helminticos polivalentes. Mesa-redonda no Nono Congresso da Sociedade Brasileira de $\mathrm{Me}$ dicina Tropical, realizado em Fortaleza, Ceará, de 4 a 7 de fevereiro de 1973 .

13. KHALIL, H. M. - Treatment of cestode infections with Radeverm. Trans R. Soc. Trop. Med. Hyg., 63: 76-8, 1969 .
14. MARTIN, L $\mathrm{K}$ \& BEAVER, $\mathrm{P}$ C Evaluation of Kato thick-smear technique for quantitative diagnosis of helminth infections. Amer. J. Trop. Med. Hyg., 18: 553-4, 1969

15. RODRIGUES DA SILVA, J., RODRIGUES, Y T., MORTEO, R , FERREIRA, L. F . \& BRASIL, H. A. - Tratamento das teníases humanas; estudo de rervisão e resultados com o "Cestocida Bayer 2353" (Yomesan) um derivado da salicilamida. Arq. brasil. med., 51: 175-178, 1961.

16. SEATON, D. R. - On the use of diclofen as a taenifuge for Taenia saginata. Ann. Trop. Med. Parasit., 54: $338-40,1960$

17. SOUZA, D. W. C., SOUZA, M. S. L . \& NEVES, J. - Ação terapêutica do mebendazole ( $R$ 17635) em pacientes poliparasitados. Resultados preliminares. Rev. Inst. Med. trop. São Paulo, 15: 30-33, 1973

18. WITTNER, M. \& TANOWITZ, H. Paromomycin therapy of human cestcdiasis with special reference to hymenolepiasis. Am. J. Trop. Med. Hyg., 20: $433-5,1971$ 